\title{
TiaochangXiaoliu decoction inhibits azomethane (AOM)/dextran sulfate sodium (DSS)-induced colorectal cancer by regulating immune response
}

\author{
Kefang Chen ${ }^{1,2}$, Beiping Zhang ${ }^{1}$, Jianjun $\mathrm{Li}^{2}$, Aizhen Pan $^{2}$, Linhui Cao ${ }^{2}$, Xiying Zhao ${ }^{1}$, Suiping Huang ${ }^{1}$, \\ Liudan Chen ${ }^{3}$
}

${ }^{1}$ Department of Spleen and Stomach Diseases, The Second Clinical College of Guangzhou University of Chinese Medicine, Guangzhou, China;

${ }^{2}$ Department of Traditional Chinese Medicine, Sun Yat-sen Memorial Hospital, Sun Yat-sen University, Guangzhou, China; ${ }^{3}$ Department of Acupuncture and Moxibustion, Sun Yat-sen Memorial Hospital, Sun Yat-sen University, Guangzhou, China

Contributions: (I) Conception and design: K Chen; (II) Administrative support: J Li, S Huang; (III) Provision of study materials or patients: K Chen, J Li, S Huang; (IV) Collection and assembly of data: B Zhang, X Zhao; (V) Data analysis and interpretation: A Pan, L Cao, L Chen; (VI) Manuscript writing: All authors; (VII) Final approval of manuscript: All authors.

Correspondence to: Suiping Huang. Department of Spleen and Stomach Diseases, The Second Clinical College of Guangzhou University of Chinese Medicine, Guangzhou, China. Email: Huichenhuayuan@126.com; Liudan Chen. Department of Acupuncture and Moxibustion, Sun Yat-sen Memorial Hospital, Sun Yat-sen University, Guangzhou, China. Email: chenliud@mail.sysu.edu.cn.

Background: TiaochangXiaoliu decoction (TXD) has an anti-tumor effect in clinical practice. We further investigated the role of TXD in colorectal cancer (CRC).

Methods: Mouse models of CRC were induced by azomethane (AOM)/dextran sulfate sodium (DSS), with sixty male C57BL/6 mice randomly divided into six groups (10 mice/group): a control group, AOM/ DSS group, TXD at low dose (L-dose) group, middle dose (M-dose) group, high dose (H-dose) group, and Celecoxib (Cel) group. The colorectum, serum, and plasma of mice in each group was collected following sacrifice to record the number of tumors. HE staining was utilized for observing pathological damage to colorectal tissues, ELISA used for detecting INF- $\gamma$, IL-2, and TNF- $\alpha$ expression in serum, and flow cytometry used for measuring the proportion of $\mathrm{CD}^{+}, \mathrm{CD}^{+}, \mathrm{CD}^{+} / \mathrm{CD}^{+}$, and $\mathrm{NK}$ cells in plasma.

Results: Compared with the control group, the AOM/DSS group showed tumor masses in the colorectum and different degrees of pathological damage in the intestine. AOM/DSS induction also resulted in an increase in INF- $\gamma$, IL-2, and TNF- $\alpha$ expression in serum, and a decrease in the percentages of CD4 $4^{+}, \mathrm{CD}^{+}$, $\mathrm{CD}^{+} / \mathrm{CD}^{+}$, and NK cells $(\mathrm{P}<0.05)$. In comparison with the AOM/DSS group, with the increase of TXD dose, the number of tumors decreased significantly, and intestinal structure and mucosal inflammatory cell infiltration also improved. Further, in comparison with the AOM/DSS group, all three doses of TXD and celecoxib caused an increase in the contents of $\mathrm{CD}^{+}, \mathrm{CD}^{+}, \mathrm{CD} 4^{+} / \mathrm{CD} 8^{+}$, and $\mathrm{NK}$ cells in plasma. In addition, in the M-dose, H-dose, and Cel groups, INF- $\gamma$, IL-2, and TNF- $\alpha$ expression showed a marked decrease, and the reduction in these two groups treated with TXD was dose-dependent.

Conclusions: TXD leads to a marked reduction in the number of tumors and inflammatory cell infiltration in CRC mice. This decoction significantly decreased the levels of INF- $\gamma$, IL-2, and TNF- $\alpha$ in serum, and increased the contents of $\mathrm{CD}^{+}, \mathrm{CD}^{+}, \mathrm{CD}^{+} / \mathrm{CD}^{+}$, and $\mathrm{NK}$ cell in the plasma of mice with AOM/DSSinduced CRC.

Keywords: TiaochangXiaoliu decoction (TXD); azomethane (AOM)/dextran sulfate sodium (DSS); colorectal cancer (CRC); immune response; tissue damage

Submitted Aug 10, 2021. Accepted for publication Sep 29, 2021.

doi: 10.21037/jgo-21-580

View this article at: https://dx.doi.org/10.21037/jgo-21-580 


\section{Introduction}

Colorectal cancer (CRC) is a common malignant tumor of the digestive tract, ranking third in incidence and mortality of cancers worldwide (1). Generally, $5-10 \%$ of CRC is hereditary, usually caused by familial adenomatous polyposis, while more than $90 \%$ are sporadic tumors (2). Most CRC patients have developed to middle and advanced stages at diagnosis, and those with advanced invasion and metastasis have a 5 -year survival rate of less than $10 \%$ and a high mortality rate, seriously threatening human health $(3,4)$. In addition, in patients with advanced CRC with metastasis, the most common distant metastasis occurs in the liver, accounting for $60 \%$ (5). Currently research on CRC mostly focus on new therapies that are simpler and more effective, or the development of new drugs to improve the overall survival rate and quality of life of CRC patients.

Chronic inflammation is closely related to the development of CRC, and "inflammation-aberrant cryptadenoma-cancer" is the basic path for the occurrence of colitis-related CRC (6). The risk of developing CRC in patients with inflammatory bowel disease (IBD) without effective treatment is 20 times higher than that of normal subjects (7). The difference in this risk suggests a pivotal role of inflammation in the development of CRC, and the control of "inflammation-cancer transformation" is of great significance for its prevention and treatment.

Traditional Chinese medicine plays an increasingly critical role in the prevention and treatment of CRC. Such as the Gegen Qinlian decoction, which has been revealed with the potential to enhance immunity and protect intestinal barrier function of CRC patients (8). Tiaochang Xiaoliu decoction (TXD) is a classical prescription commonly utilized for the clinical treatment of cancers, which is composed of nine herbs including Dang Shen (Radix Codonopsis), Bai Zhu (Rhizoma Atractylodis Macrocephalae), Huang Qi (Radix Astragali seu Hedysari), Yi Yi Ren (Semen Coicis), Dou Kou Ren (Fructus Ammomi Rotundus), Bai Hua She She Cao (Herba Hedyotis), E Zhu (Rhizoma Curcumae), San Qi (Radix Notoginseng), and Zhi Gan Cao (Radix Glycyrrhizae Preparata). While studies have shown that TXD can reduce the recurrence rate of colorectal adenoma (9), the mechanism remains unclear. In order to explore the underlying mechanism, azomethane $(\mathrm{AOM}) /$ dextran sodium sulfate (DSS) administration was utilized to induce a mouse model for the investigation of the effect of TXD on CRC and its mechanism, potentially providing a new treatment for the disease. We present the following article in accordance with the ARRIVE reporting checklist (available at https://dx.doi.org/10.21037/jgo-21-580).

\section{Methods}

\section{Experimental animals}

Prior to formal experimentation, 60 healthy SPF male C57BL/6 mice aged 6 weeks old and weighing 18-22 g were obtained from the Guangdong Medical Laboratory Animal Center and adaptively fed for 14 days. This study was approved by the Animal Ethics Committee of Guangzhou University of Chinese Medicine (No. 2021000617), in compliance with Experimental Animal Center, Guangzhou University of Chinese Medicine guidelines for the care and use of animals.

\section{Experimental drugs}

TXD composed of Radix Codonopsis $15 \mathrm{~g}$, Rhizoma Atractylodis Macrocephalae $15 \mathrm{~g}$, Radix Astragali seu Hedysari 15 g, Semen Coicis 30 g, Fructus Ammomi Rotundus 10 g, Herba Hedyotis 30 g, Rhizoma Curcumae 15 g, Radix Notoginseng $10 \mathrm{~g}$, and Radix Glycyrrhizae Preparata $5 \mathrm{~g}$, was obtained from the pharmacy of the Sun Yatsen Memorial Hospital, Sun Yat-sen University, and was identified by appraisers in the pharmacy department of the Hunan Cancer Hospital. The materials were soaked in warm water $3-5 \mathrm{~cm}$ above the surface of the drugs for $1 \mathrm{~h}$, then boiled in high heat and fried in low heat for $30 \mathrm{~min}$, following by a filtration step when the medicines were still at a high temperature. The residue was decocted twice as a previous procedure, and the solution from these three treatments was mixed and filtered. Finally, in a constant temperature water bath, the concentration of crude drugs was adjusted to $3.6 \mathrm{~g} / \mathrm{mL}$, and the decoction was stored at $4{ }^{\circ} \mathrm{C}$ for later use.

\section{Establishment of AOM-DSS model and grouping}

The mice were randomly divided into six groups of 10 . In the control group, mice were treated with the same amount of solvent. In the AOM/DSS group, mice were intraperitoneally injected with AOM $(10 \mathrm{mg} / \mathrm{kg}$; Sigma, USA) on the first day of the first week, then fed with drinking water supplemented with $2.5 \%$ DSS (Beijing Coolaber, China) for 1 week, followed by normal drinking water for 2 weeks, with 3 weeks as a cycle, which was 
repeated three times (10). On the basis of AOM/DSS treatment, drug intervention was performed in the low $(\mathrm{L})$, middle $(\mathrm{M})$, and high $(\mathrm{H})$ dose groups according to the reference (9). Specifically, at the same time as the intraperitoneal injection of $\mathrm{AOM}$, mice of the $\mathrm{L}, \mathrm{M}$, and $\mathrm{H}$ groups were intragastrically administered with TXD for 10 weeks at a dosage of $0.07 \mathrm{~mL} /$ day/mouse, $0.14 \mathrm{~mL} /$ day/mouse, $0.28 \mathrm{~mL} /$ day/mouse, respectively. In the Celecoxib (Cel) group, at the same time as the intraperitoneal injection of $\mathrm{AOM}$, mice were intragastrically administered with celecoxib $(20 \mathrm{mg} / \mathrm{kg} /$ day) (Sun Yatsen Memorial Hospital, Sun Yat-sen University, China) for 10 weeks. Mice in the control and AOM/DSS groups were sacrificed by cervical dislocation on the last day of the $9^{\text {th }}$ week, and those in the other four groups on the last day of the $10^{\text {th }}$ week. The condition of tumors in mice was observed, and the number of tumors was recorded. Colorectal, serum, and plasma samples were collected from the mice for subsequent index assays.

\section{HE staining}

Mouse colons were fixed in $10 \%$ formalin and dehydrated with different concentrations of ethanol, followed by paraffin embedding. The embedded colons were cut into 5 - $\mu \mathrm{m}$ sections using a microtome and placed in xylene to dissolve paraffin, then soaked with ethanol from high to low concentrations, and finally placed in deionized water. Hematoxylin staining solution was utilized for staining, and hydrochloric acid-ethanol solution for differentiation. Subsequently, the sections were rinsed in water for $20 \mathrm{~min}$ for bluing, following which they were rapidly dehydrated and mounted after being stained with eosin stain. Mouse colon tissues were observed using an ordinary light microscope (11).

\section{ELISA}

According to the instructions of INF- $\gamma$, IL-2, and TNF- $\alpha$ ELISA kits (Wuhan Colorful Gene Biological Technology Co., LTD., China), the standards were gradient diluted to draw the standard curve. Mouse serum was added to 96-well plates coated with anti-INF- $\gamma$, IL-2, or TNF- $\alpha$ antibodies, respectively, along with the standards at different concentrations. Following a $30 \mathrm{~min}$ reaction, chromogenic substrate was added, and subsequently a reaction termination solution was also added. On completion of the rinsing step, the 96-well plates were placed in a microplate reader for detecting the absorbance value $(450 \mathrm{~nm})$, thus calculating the levels of INF- $\gamma$, IL-2, and TNF- $\alpha$ in the serum of each mouse (12).

\section{Flow cytometry}

The cTfh cell surface molecules were recognized by the following anti-mouse monoclonal antibodies: CD3 PerCP, CD4 FITC, CD8 PE, and CD (16+56)-PE, and CD3+, $\mathrm{CD}^{+}, \mathrm{CD}^{+}$, and $\mathrm{NK}$ cells and the corresponding isotype control antibodies were identified. The specific steps were as follows: First, $10 \mu \mathrm{L}$ of antibody was added into $100 \mu \mathrm{L}$ of anticoagulant and placed in the dark at ambient temperature for $10 \mathrm{~min}$ after being fully mixed on the shaker. Then, $500 \mu \mathrm{L}$ of red blood cell lysate was added, mixed, and placed in the dark at ambient temperature for $20 \mathrm{~min}$. Next, the supernatant was aspirated after centrifugation at 3,000 $\mathrm{r}$ for $5 \mathrm{~min}$, followed by an addition of $500 \mu \mathrm{L}$ of buffer. After being mixed, the sample was detected by flow cytometry for determining the percentage of $\mathrm{CD}^{+}, \mathrm{CD}^{+}, \mathrm{CD}^{+} / \mathrm{CD}^{+}$, and NK cells in mouse plasma (13).

\section{Statistical analysis}

The experimental data were statistically analyzed using SPSS 22.0 software. $T$-test was adopted for comparison between groups, while one-way analysis of variance was used for comparison among multiple groups. The results were expressed as mean \pm standard deviation $(\mathrm{SD})$, and a statistically significant difference was suggested if $\mathrm{P}<0.05$.

\section{Results}

\section{TXD reduces AOM/DSS-induced CRC}

Colorectal tissues were collected from mice in each group, and no tumors were observed in the control group, while different numbers of tumor masses were observed in the tissues of the other five groups (Figure 1A). Specifically, in comparison with the AOM/DSS group, the number of tumors in the L-dose, $\mathrm{M}$-dose, $\mathrm{H}$-dose, and Cel groups was significantly decreased, and the decrease in the groups treated with TXD was concentration-dependent (Figure 1B).

HE staining results (Figure 1C) revealed normal structure of the colon and no inflammatory cell infiltration in the control group. However, in the AOM/DSS group, darker nuclear staining was found in glandular epithelial cells of tumor tissue in the colonic mucosa, indicating well- 
A
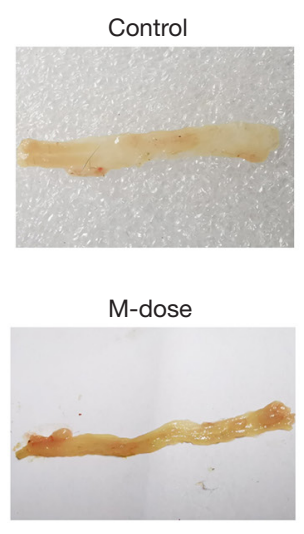
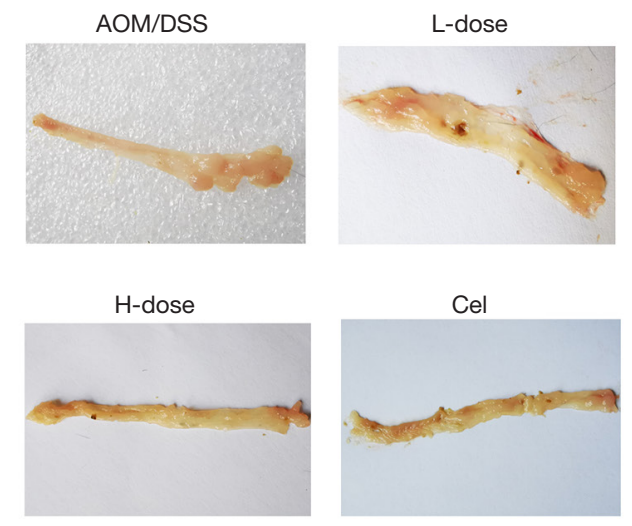

B

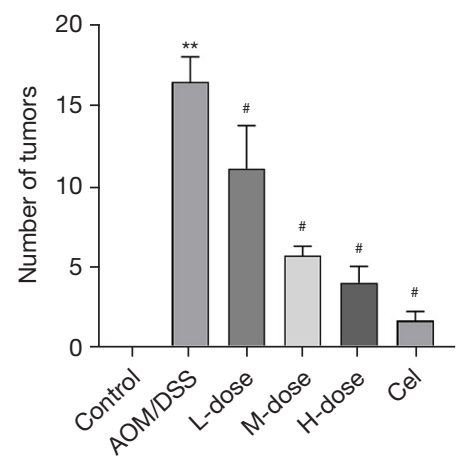

C

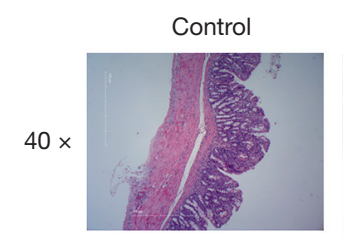

AOM/DSS
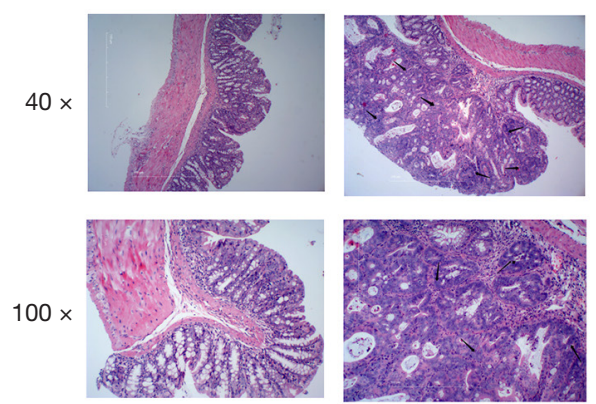
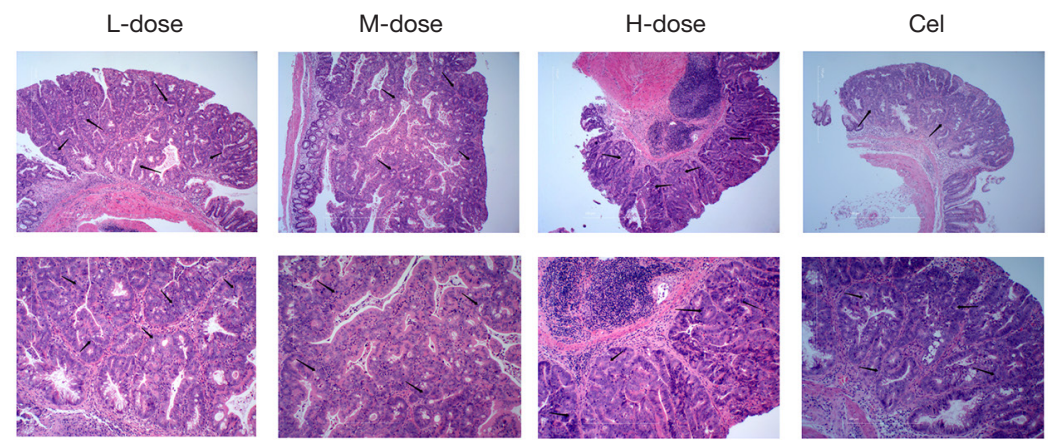

Figure 1 Effect of Tiaochang Xiaoliu Decoction on AOM/DSS-induced colorectal cancer. (A) Colorectal tissues of mice in each group; (B) number of tumors in the rectum of mice in each group; (C) HE staining for observing the pathological damage to colorectal tissues of mice in each group. **, $\mathrm{P}<0.01$ vs. control group;, $\mathrm{P}<0.05$ vs. ADM/DSS group. ADM/DSS, azomethane/dextran sulfate sodium.

differentiated adenocarcinoma. Additionally, increasing neovascularization at the edges of tumors, and inflammatory cell infiltration in mucosa and submucosa of some colonic tissues could be seen in the AOM/DSS group. The condition was similar in the L-dose group and M-dose group, with tumor tissue in colonic mucosa, and a small amount of necrotic tissue in the middle of tumor. The $\mathrm{H}$-dose group showed tumor tissue in colonic mucosa, but with less atypia, and the structural change of colon was not obvious in most cases. In the Cel group, tumor tissue was also observed in colonic mucosa and the glands were arranged neatly in most cases, only presenting as atypical hyperplasia. Additionally, the mice of the Cel group showed inflammatory cell infiltration in mucosa and submucosa.

Collectively, TXD could effectively inhibit CRC induced by AOM/DSS in a dose-dependent manner.

\section{TXD Improves AOM/DSS-Induced inflammation in mice}

ELISA results (Figure 2) showed that IL-2, INF- $\gamma$, and TNF- $\alpha$ expression in the serum of mice in the AOM/DSS group were significantly higher than those in the control group. Further, in comparison with the AOM/DSS group, their expression showed a marked decrease in M-dose, $\mathrm{H}$-dose and Cel groups in a concentration-dependent manner. Collectively, TXD could significantly inhibit the inflammation in mice induced by AOM/DSS.

\section{TXD promotes the expression of immune cells in mice with colitis-relatedCRC}

As shown in the flow cytometry results (Figure $3 A, 3 B$ ), in comparison with the control group, the percentages of $\mathrm{CD}^{+}, \mathrm{CD}^{+}, \mathrm{CD}^{+} / \mathrm{CD}^{+}$and $\mathrm{NK}$ cells in the plasma of 

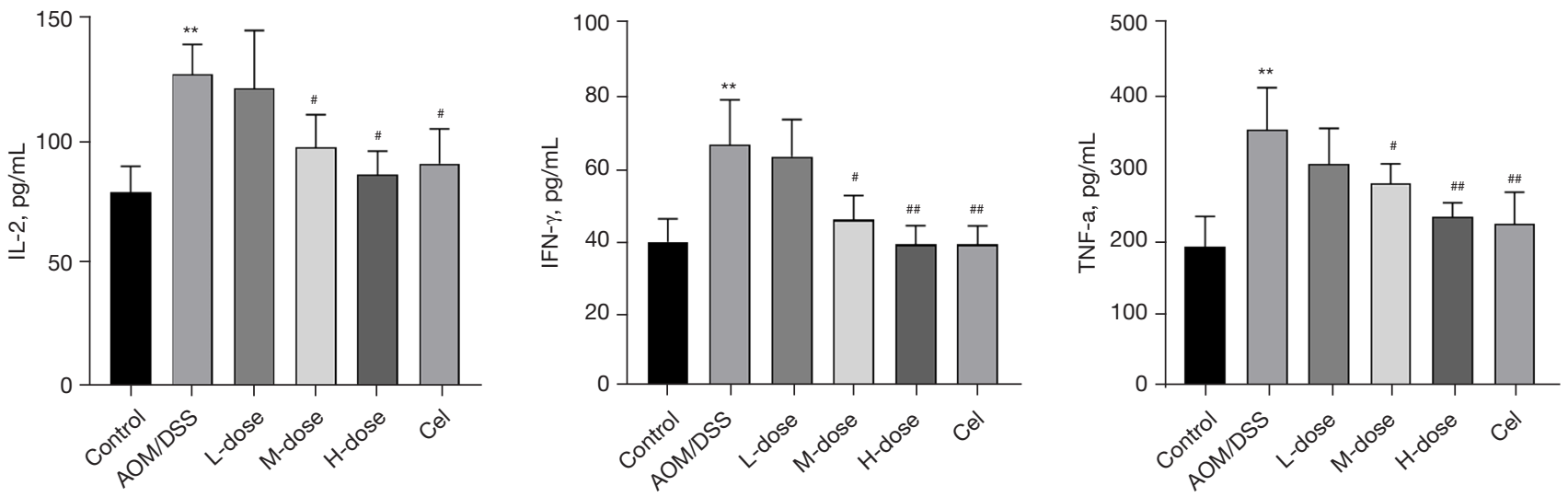

Figure 2 Effect of Tiaochang Xiaoliu decoction on AOM/DSS-induced inflammation. ELISA was utilized to detect the INF- $\gamma$, IL-2, and TNF- $\alpha$ expression in the serum of mice in each group. **, $\mathrm{P}<0.01$ vs. control group; ${ }^{*}, \mathrm{P}<0.05$ and ${ }^{\#}, \mathrm{P}<0.01$ vs. ADM/DSS group. ADM/ DSS, azomethane/dextran sulfate sodium.

A
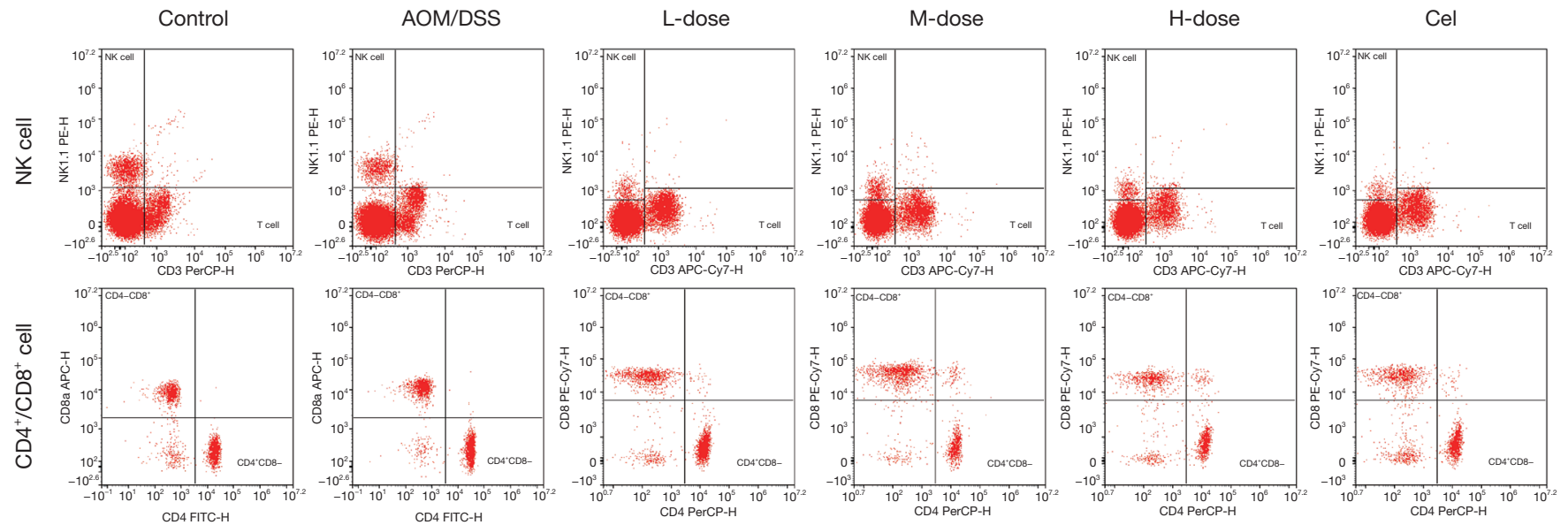

B
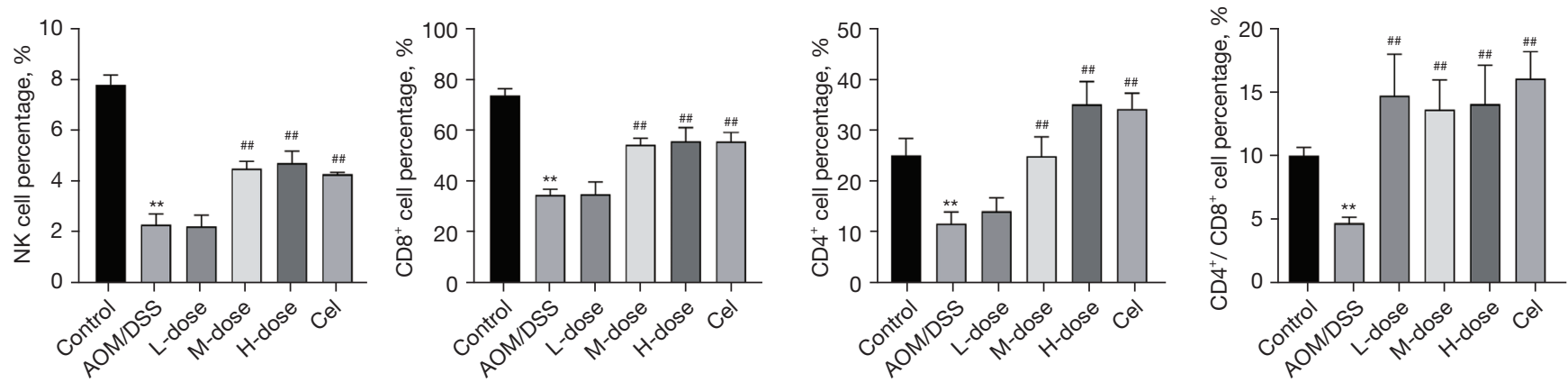

Figure 3 Effect of Tiaochang Xiaoliu decoction on immune cells in mice with colitis-related colorectal cancer. (A) Flow cytometry was utilized to detect the changes of NK cells and $\mathrm{CD}^{+} / \mathrm{CD}^{+}$cells in the plasma of cells; (B) rate of cell apoptosis. ${ }^{* *}, \mathrm{P}<0.01$ vs. control group; \#, $\mathrm{P}<0.01$ vs. ADM/DSS group. ADM/DSS, azomethane/dextran sulfate sodium. 
the AOM/DSS group were markedly decreased. Further, in comparison with the AOM/DSS group, all three doses of TXD and celecoxib caused an increase in the $\mathrm{CD} 4^{+} / \mathrm{CD} 8^{+}$ cell content in plasma, and the contents of $\mathrm{CD} 4^{+}, \mathrm{CD} 8^{+}$and NK cells in plasma significantly increased in the M-dose, $\mathrm{H}$-dose and Cel groups, with the increase in the M-dose and $\mathrm{H}$-dose groups concentration-dependent. No significant difference was identified between the H-dose and Cel groups.

Collectively, TXD could effectively improve immune function which was inhibited by AOM/DSS.

\section{Discussion}

CRC is a malignant tumor with a high frequency and yearly increasing incidence in China (14). Even in developed countries with advanced medical technology, the disease remains a serious threat to human health (15). Studies have found that the pathogenesis of CRC is closely related to chronic intestinal inflammation, which induces tumor transformation of colonic epithelial cells and promotes cell proliferation and invasion, leading to the infiltration of immune cells and soluble mediators, and ultimately providing a favorable microenvironment for tumor development $(16,17)$. Accumulating evidence suggests that patients with IBD have a high risk of CRC $(18,19)$, and the relief of intestinal inflammation has been considered a critical strategy for the prevention and treatment $(20,21)$. From the perspective of traditional Chinese medicine, deficiency of spleen qi is the cause of the occurrence and development of CRC, and the subsequent internal retention of water results in phlegm-dampness and stagnant toxicity in the colon, and consequently cancer. Deficiency of spleen qi and kidney was reported as the pathological base of malignant tumors, and the combination of phlegm stasis and heat-toxicity is an important pathogenesis of CRC (22). Emodin could suppressed carcinogenesis-associated intestinal inflammation and prevents AOM/DSS-induced intestinal tumorigenesis and progression (23). Quercetin was found with the similar functions on AOM/DSS-induced CRC (24). All above indicated that the anti-inflammatory agents have the potential for the treatment and prevention of CRC. TXD is a special prescription for CRC, with effects of invigorating the spleen and supplementing qi, eliminating dampness, removing blood stasis, and detoxicating. Modern pharmacology suggests the prescription of invigorating spleen and supplementing invigorating qi can achieve antitumor effects by improving the immune status of the body and epithelial-mesenchymal transition of tumors $(25,26)$.
The prescription of blood circulation promotion for removing blood stasis and of detoxicating, can inhibit tumor angiogenesis and improve the immune status of the body, inhibiting tumors (27).

The active components of Radix Codonopsis, such as saponin and polysaccharide, are effective in improving inflammation and immune regulation (28,29). Polyphenols, which are the active ingredient in Semen Coicis, can enhance immunomodulation, anti-tumor, anti-oxidation, and anti-inflammation (29). The active ingredients in Radix Astragali seu Hedysari, such as polysaccharides, saponins, and flavonoids, function in immune regulation and in the inhibition of tumors. For example, Astragalus polysaccharides could significantly inhibit S180 sarcoma by increasing the expression of $\mathrm{CD} 4^{+} \mathrm{T}$ cells in peripheral blood while decreasing that of $\mathrm{CD} 8^{+} \mathrm{T}$ cells, and upregulating IL-2 expression while down-regulating IL-4 expression, indicating that its effect was achieved through regulating immunity (30). Chou et al. (31) applied Qingshu Yiqi decoction with Rhizoma Atractylodis Macrocephalae as the major component, to treat mice with Lewis lung carcinoma, and found it prevented weight loss and reduced the secretion of IL1 $\beta$, IL 6 and TNF- $\alpha$ by macrophages, exhibiting an anti-tumor and anti-inflammation effect. Polysaccharides of Herba Hedyotis were reported to improve immune function and inhibit CRC cell HT29 proliferation in immunosuppressed mice $(32,33)$, and through the JAK/STAT pathway, Rhizoma Curcumae could effectively inhibit tumor growth and promote the development and differentiation of the spleen in rats with lung cancer (34). Radix Notoginseng oral liquid combined with pneumatic compression therapy improved plasma prothrombin time, activated partial thromboplastin time, and stabilized platelet levels, achieving improvement in the blood hypercoagulable state of cancer patients, and reduction of the incidence of lower extremity deep venous thrombosis (35). Chen et al. (36) found that glycyrrhiza polysaccharide in Radix Glycyrrhizae Preparata could accelerate the growth of mice and improve immune function by promotion the function of organs such as the spleen and thymus. In addition, Radix Glycyrrhizae Preparata was seen to contain many flavonoids, which have an inhibitory effect on the proliferation of hepatoma cells, leukemia cell HL-60, and human fibrosarcoma cell HT-1080 (37).

The AOD/DSS model has been widely accepted, since AOD could induce the DNA mutations and DSS could induce intestinal inflammation (38). In this model the pathological processes leading to cancer are similar to 
those in humans CRC pathogenesis, including mucosal inflammation, and epithelial hyperplasia and dysplasia (39). In this study, a mouse model induced by AOM/DSS was adopted for investigation of the effects of TXD on CRC, to provide a reference basis for clinical treatment. The results showed that TXD reduced the number of tumors and the degree of inflammatory cell infiltration, decreased TNF- $\alpha$, INF- $\gamma$, and IL-2 expression in serum, and increased the content of $\mathrm{CD}^{+}, \mathrm{CD}^{+}, \mathrm{CD}^{+} / \mathrm{CD}^{+}$, and $\mathrm{NK}$ cells in plasma in a concentration-dependent manner, indicating that TXD was effective for CRC.

\section{Conclusions}

In summary, TXD leads to marked reduction in the number of tumors and inflammatory cell infiltration in mouse models of CRC, and a significant increase in the content of immune cells in plasma in a concentration-dependent manner. The efficacy of TXD may be related to the nine kinds of active ingredients in the prescription, and its specific mechanism remains to be further studied.

\section{Acknowledgments}

Funding: None.

\section{Footnote}

Reporting Checklist: The authors have completed the ARRIVE reporting checklist. Available at https://dx.doi. org/10.21037/jgo-21-580

Data Sharing Statement: Available at https://dx.doi. org/10.21037/jgo-21-580

Conflicts of Interest: All authors have completed the ICMJE uniform disclosure form (available at https://dx.doi. org/10.21037/jgo-21-580). The authors have no conflicts of interest to declare.

Ethical Statement: The authors are accountable for all aspects of the work in ensuring that questions related to the accuracy or integrity of any part of the work are appropriately investigated and resolved. Experiments were performed under a project license (No. 2021000617) granted by the Animal Ethics Committee of Guangzhou University of Chinese Medicine, in compliance with Experimental Animal Center, Guangzhou University of Chinese Medicine guidelines for the care and use of animals.

Open Access Statement: This is an Open Access article distributed in accordance with the Creative Commons Attribution-NonCommercial-NoDerivs 4.0 International License (CC BY-NC-ND 4.0), which permits the noncommercial replication and distribution of the article with the strict proviso that no changes or edits are made and the original work is properly cited (including links to both the formal publication through the relevant DOI and the license). See: https://creativecommons.org/licenses/by-nc-nd/4.0/.

\section{References}

1. Torre LA, Bray F, Siegel RL, et al. Global cancer statistics, 2012. CA Cancer J Clin 2015;65:87-108.

2. Calin GA, Sevignani C, Dumitru CD, et al. Human microRNA genes are frequently located at fragile sites and genomic regions involved in cancers. Proc Natl Acad Sci U S A 2004;101:2999-3004.

3. Siegel R, Naishadham D, Jemal A. Cancer statistics, 2012. CA Cancer J Clin 2012;62:10-29.

4. Marquardt JU, Fischer K, Baus K, et al. Sirtuin-6dependent genetic and epigenetic alterations are associated with poor clinical outcome in hepatocellular carcinoma patients. Hepatology 2013;58:1054-64.

5. Mitchell PS, Parkin RK, Kroh EM, et al. Circulating microRNAs as stable blood-based markers for cancer detection. Proc Natl Acad Sci U S A 2008;105:10513-8.

6. Shawki S, Ashburn J, Signs SA, et al. Colon Cancer: Inflammation-Associated Cancer. Surg Oncol Clin N Am 2018;27:269-87.

7. Lasry A, Zinger A, Ben-Neriah Y. Inflammatory networks underlying colorectal cancer. Nat Immunol 2016;17:230-40.

8. Li Y, Li ZX, Xie CY, et al. Gegen Qinlian decoction enhances immunity and protects intestinal barrier function in colorectal cancer patients via gut microbiota. World J Gastroenterol 2020;26:7633-51.

9. Li Y, Zhong CL, Yang SP. Effect of TiaochangXiaoliu Formulaon the Recurrence Rate of Patients with Colorectal Adenoma and the Expression of Beclinl,pand Cox-2 in Colon Tissue. Journal of Traditional Chinese Medicine 2021;62:424-7.

10. Liu L, Gao H, Wen T, et al. Tanshinone IIA attenuates $\mathrm{AOM} / \mathrm{DSS}$-induced colorectal tumorigenesis in mice via inhibition of intestinal inflammation. Pharm Biol 2021;59:89-96. 
11. Seetha A, Devaraj H, Sudhandiran G. Effects of combined treatment with Indomethacin and Juglone on AOM/DSS induced colon carcinogenesis in Balb/c mice: Roles of inflammation and apoptosis. Life Sci 2021;264:118657.

12. Dai GL, Yang XY, Chen S, et al. Inhibitory Effects of DahuangZhechong Pill on AOM/DSS Induced Colitisassociated Cancer in Mice. Pharmaceutical and Clinical Research 2021;29:1-6.

13. Kienzl M, Hasenoehrl C, Valadez-Cosmes P, et al. IL-33 reduces tumor growth in models of colorectal cancer with the help of eosinophils. Oncoimmunology 2020;9:1776059.

14. Siegel R, Ma J, Zou Z, et al. Cancer statistics, 2014. CA Cancer J Clin 2014;64:9-29.

15. Hawk ET, Limburg PJ, Viner JL. Epidemiology and prevention of colorectal cancer. Surg Clin North Am 2002;82:905-41.

16. Park JH, Kim JK. Pristimerin, a naturally occurring triterpenoid, attenuates tumorigenesis in experimental colitis-associated colon cancer. Phytomedicine 2018;42:164-71.

17. Pejin B, Iodice C, Bogdanović G, et al. Stictic acid inhibits cell growth of human colon adenocarcinoma HT-cells. Arabian Journal of Chemistry 2017;10:S1240-2.

18. Malhotra A, Phatharacharukul P, Thongprayoon C. Risk factors for 90-day readmission in veterans with inflammatory bowel disease-Does post-discharge followup matter? Mil Med Res 2018;5:5.

19. Zhou D, Jin J, Liu Q, et al. PPAR $\delta$ agonist enhances colitis-associated colorectal cancer. Eur J Pharmacol 2019;842:248-54.

20. Pejin B, Jovanović KK, Mojović M, et al. New and highly potent antitumor natural products from marine-derived fungi: covering the period from 2003 to 2012. Curr Top Med Chem 2013;13:2745-66.

21. Zhao Q, Bi Y, Zhong J, et al. Pristimerin suppresses colorectal cancer through inhibiting inflammatory responses and $W n t / \beta$-catenin signaling. Toxicol Appl Pharmacol 2020;386:114813.

22. Wei C, Yuan GQ, Chang LP, et al. Improving syndromes-A new strategy for tumor treatment. Journal of Traditional Chinese Medicine 2014;55:555-7.

23. Zhang Y, Pu W, Bousquenaud M, et al. Emodin Inhibits Inflammation, Carcinogenesis, and Cancer Progression in the AOM/DSS Model of Colitis-Associated Intestinal Tumorigenesis. Front Oncol 2021;10:564674.

24. Lin R, Piao M, Song Y, et al. Quercetin Suppresses AOM/DSS-Induced Colon Carcinogenesis through
Its Anti-Inflammation Effects in Mice. J Immunol Res 2020;2020:9242601.

25. Dong QH, Jin JY, Zhu J, et al. Effect of Yiqijianpihuaji Recipe on immune function and quality of life of patients with advanced colon cancer. Zhejiang Journal of Integrated Traditional Chinese and Western Medicine 2019;29:994-6.

26. Wu J, Chen Yz, Zhang X, et al. Inhibitory Effect and Mechanism of Yiqijianpihuayu Recipe Combined with -FU on Gastric Cancer. Chinese Journal of Experimental Traditional Medical Formulae 2020;26:65-72.

27. Tang ZN, Luo YP, Ge F, et al. Researchprogress on the mechanism of anti-tumor Chinese herbal medicine by promoting blood circulation and removing blood stasis. China Pharmacy 2016;27:1146-9.

28. Chu X, Liu XJ, Qiu JM, et al. Effects of Astragalus and Codonopsis pilosula polysaccharides on alveolar macrophage phagocytosis and inflammation in chronic obstructive pulmonary disease mice exposed to PM2.5. Environ Toxicol Pharmacol 2016;48:76-84.

29. Zou YF, Chen XF, Malterud KE, et al. Structural features and complement fixing activity of polysaccharides from Codonopsis pilosula Nannf. var. modesta L.T.Shen roots. Carbohydr Polym 2014;113:420-9.

30. Zou PW, Zhao CJ, Li P, et al. Effects of Astragalus polysaccharides on Anti-Ssarcoma and immunological regulation. Acta AcademiaeMedicinae Zunyi 2012;35:17-20.

31. Chou YJ, Kuo CY, Chen PY, et al. Oral administration of Qing-Shu-Yi-Qi-Tang reduce lung cancer-induced CRChexia in mice. African Journal of Pharmacy and Pharmacology 2012;6:84-91.

32. Qu JY, Tian M, He JH, et al. Immunomodulatory effect of polysaccharide from HerbaHedyotison immunosuppressed mice. Journal of Chinese Medicinal Materials 2015;38:1942-5.

33. Lin J, Wei L, Shen A, et al. Hedyotis diffusa Willd extract suppresses Sonic hedgehog signaling leading to the inhibition of colorectal cancer angiogenesis. Int J Oncol 2013;42:651-6.

34. Zhao MZ, Liu HY, Zhang XL. Inhibitory effect of curcuma zedoaria on lung cancer in rats and its effect on JAK-STAT signaling pathway.Modern Journal of Integrated Traditional Chinese and Western Medicine 2020;29:3658-61+3665.

35. Liu ZZ. Clinical study of Radix Notoginseng oral liquid combined with pneumatic compression therapy in the prevention of lower extremity deep venous thrombosis in cancer patients. China Practical Medical 2019;14:123-24. 
36. Chen J, Zhu XQ, Yang L, et al. Effect of Glycyrrhiza uralensis Fisch polysaccharide on growth performance and immunologic function in mice in Ural City, Xinjiang. Asian Pac J Trop Med 2016;9:1078-83.

37. Cui YR, Li DF, Ju B, et al. Antitumor effects of four licoflavones in vitro. Food Science and Technology 2010;35:88-92.

38. Tanaka T, Kohno H, Suzuki R, et al. A novel inflammation-related mouse colon carcinogenesis model induced by azoxymethane and dextran sodium sulfate. Cancer Sci 2003;94:965-73.

39. Parang B, Barrett CW, Williams CS. AOM/DSS

Model of Colitis-Associated Cancer. Methods Mol Biol 2016;1422:297-307.

(English Language Editor: B. Draper)
Cite this article as: Chen $\mathrm{K}$, Zhang $\mathrm{B}$, Li J, Pan A, Cao L, Zhao X, Huang S, Chen L. TiaochangXiaoliu decoction inhibits azomethane (AOM)/dextran sulfate sodium (DSS)induced colorectal cancer by regulating immune response. J Gastrointest Oncol 2021;12(5):2223-2231. doi: 10.21037/jgo-21580 